\title{
Article \\ Explanation and Application of the Evolving Contact Traction Fields in Shallow Foundation Systems
}

\author{
Adam G. Taylor * (i) and Jae H. Chung \\ Lawrence Livermore National Laboratory, Livermore, CA 94550, USA; chung29@llnl.gov \\ * Correspondence: taylor265@1lnl.gov
}

\begin{abstract}
The present paper provides a qualitative discussion of the evolution of contact traction fields beneath rigid shallow foundations resting on granular materials. A phenomenological similarity is recognized in the measured contact traction fields of rigid footings and the bases of sandpiles. This observation leads to the hypothesis that the stress distributions are brought about by the same physical phenomena, namely the development of arching effects through force chains and mobilized intergranular friction. A set of semi-empirical equations are suggested for the normal and tangential components of this contact traction based on past experimental measurements and phenomenological assumptions of frictional behaviors at the foundation system scale. These equations are then applied to the prescribed boundary conditions for the analysis of the settlement, resistance, and stress fields in supporting granular materials beneath the footing. A parametric sensitivity study is performed on the proposed modelling method, highlighting solutions to the boundary-value problems in an isotropic, homogeneous elastic half-space.
\end{abstract}

Keywords: shallow foundations; soil-structure interaction; contact pressure distribution; elasticity

check for

updates

Citation: Taylor, A.G.; Chung, J.H. Explanation and Application of the Evolving Contact Traction Fields in Shallow Foundation Systems. Geotechnics 2022, 2, 91-113. https:// doi.org/10.3390/geotechnics2010004

Academic Editor: Kenneth Gavin

Received: 18 November 2021

Accepted: 28 December 2021

Published: 14 January 2022

Publisher's Note: MDPI stays neutral with regard to jurisdictional claims in published maps and institutional affiliations.

Copyright: () 2022 by the authors Licensee MDPI, Basel, Switzerland. This article is an open access article distributed under the terms and conditions of the Creative Commons Attribution (CC BY) license (https:// creativecommons.org/licenses/by/ $4.0 /)$.

\section{Introduction}

It is well known that the contact pressures which develop between a surface footing and soil body are in general not uniform. Many studies, both empirical [1-5] and analytical [5-9], have suggested that the distribution of normal pressure at contact is saddle-shaped, exhibiting a pressure dip at the center of the load. The peak values occur at some distance from the center, which appears to be dependent on intrinsic multiscale soil properties and evolves with applied loading criteria. For a given case, there also exists a tangential traction field at the interface between a normally loaded foundation and a soil body due to friction and the horizontal expansion of material beneath a compressive load $[2,6,7,10,11]$. These facts are often neglected in practical engineering calculations; however, it is a major premise of the present paper that the spatial distributions and directions of the traction fields developed during soil-structure interaction are non-negligible when assessing structural settlement and resulting stress-equilibrium states. A priori knowledge of the nonlinear development of these traction fields, applied as boundary conditions in analysis, allows for the approximate modeling of mechanical behaviors reflecting the highly nonlinear and heterogeneous nature of granular materials. This is true even within the context of continuum models with simple (linear) constitutive relationships.

The literature contains several examples of analyses that shed some light upon the mechanisms leading to the development of the saddle-shaped normal traction distributions. The defining factor is the location of the peak magnitude of contact pressure and its evolution over various loading cases. Empirical measurements show that these peak locations tend to begin towards the edge of a footing for initial small loads, but progress towards the center as the load magnitude increases ([2,3]). Mathematical models that describe this phenomena come in at least three distinct flavors: 
(i) By treating the problem in the context of continuum elastoplasticity, researchers have described this phenomena through the presence of plastic yielding $[5,12,13]$. Localized plasticity is initialized at various loading stages due to the arbitrarily large edge pressures present in the case of a purely elastic rigid punch [14,15].

(ii) Using a simpler two-dimensional model containing only springs, Kerr [9] was able to replicate this behavior by extending the classic Winkler model [16] by adding a second layer of normal springs separated by a shear layer; in this way the phenomena is mechanically linked with the concept of shear resistance. In this model, evolution of the peak location in the normal traction is related to the size of the footing and not necessary the magnitude of the load, although both of these quantities obviously contribute to the magnitudes of pressure applied to the underlying soil.

(iii) Smoltczyk [8] developed an analytical expression for the normal contact traction based on an assumed probability distribution that force be transferred to the ground between the edge and center of the footing. This represents the statistical likelihood of force propagation along granular contact chains.

Although relatively successful in a qualitative representation of the empirical phenomena, these models remain somewhat unsatisfactory for application to geotechnical problems; they do not explicitly contain concepts or parameters specific to soil bodies. They represent the complex mechanical behavior resulting from the discrete interaction of individual grains through simplified macroscopic assumptions. Although these types of simplifying assumptions are almost always necessary when modeling mechanical properties of soil, none of them are quite capable of explaining the phenomena that they represent. This paper attempts to lay out an explanatory hypothesis for this pressure dip phenomena based on the underlying mechanisms of a granular material, while remaining within the context of simplified continuum calculations.

Furthermore, the distribution of shear traction present at the contact interface of a shallow foundation has received less attention than the normal distribution. This is to be expected, since the normal behavior is the most relevant to the serviceability and strength of normally loaded foundations. However, it is clear from the presence of friction alone that the shear components of traction at the contact interface will be nonzero even in the case of purely normal and symmetric loads; this is granted even in the classical theories of contact with friction [17]. These components of traction must be considered in any analysis where accuracy in the local stress tensor is desired. Limited empirical measurements have been made in an attempt to quantify the shear traction $[2,10,11,18]$; similarly, there exist rudimentary semi-empirical equations that aim to approximate the distribution [19]. However, these have rarely been considered to prescribe the boundary conditions in the analysis of contact problems, and no general method exists at this time for the prediction of the spatial distribution of shear traction.

More research is required to provide a comprehensive account of the foundation contact traction fields, even for the simplest cases of homogeneous and cohesionless sands. Recent work [20,21] has advocated for the inclusion of empirical measurements in those boundary value problems of elasticity that have historically been applied to the analysis of shallow foundation systems. While it is often not feasible to empirically assess the stress fields present within the internal fabric of a real soil body, the surface traction conditions are measurable in an experimental or field domain. A simple argument from continuity shows that if realistic surface (boundary) values are enforced in a continuum-based model, the resulting stress analysis will likely be more reflective of the real physical state of the granular material, at least in the neighborhood of the boundary.

The goal of the present study is to formulate a generalized understanding of the development of contract traction distributions in the context of elasticity by providing a qualitative description based on a strong analogy between the measured foundation fields and the stress states at the bases of static sandpiles, e.g., those measured and presented in [22-24]. Furthermore, this paper seeks to motivate future research on this topic by showcasing the usefulness of accurate empirically informed boundary conditions within 
our analytical models. Based on past empirical measurements and qualitative observations of the evolution of combined normal and tangential traction fields, a parametric study is performed for prescribed Neumann boundary conditions on a homogeneous, isotropic, linear-elastic half-space.

\section{Results}

\subsection{Contact Traction, Mobilized Shear Resistance, and the Static Sandpile}

The evolution of the pressure-dip in the normal contact traction distribution of a footing on dry sand is exhibited most prominently in a laboratory experiment performed by Murzenko [3]. That author measured the spatial distributions of normal contact pressures beneath the center and diagonal lines of a relatively rigid square ram on a compacted, dry sand under increasing and repeated loads (Figure 1). The nature of these distributions appears to depend on the magnitude of the external load, as well as the loading history of the foundation system. Furthermore, this experiment reported considerable redistribution of pressure under cyclic loading, where pressure peaks appeared closer to the center of the load for the same load magnitudes after reloading. The site-scale measurements of Muhs and Bub [2] provides evidence that there exists unique distributions of tangentially acting traction that correspond and evolve alongside the normal distributions. The experiment of Lazebnik and Smirnov [1] suggests that this type of normal pressure distribution extends to some degree into the interior of the sand body, but ultimately becomes parabolic at some depth away from the loaded footing. The precise distributions that develop for a given loading scenario will depend on various inherent soil properties, including mobilized shear resistance, internal friction parameters, intergranular force chain development, and arching effects. As it stands, no comprehensive theory exists that the engineer can use to predict a pressure distribution for a given foundation system based explicitly on the properties of the granular material (intergranular friction, loading history/conditions, and the arrangement/properties of individual particles).

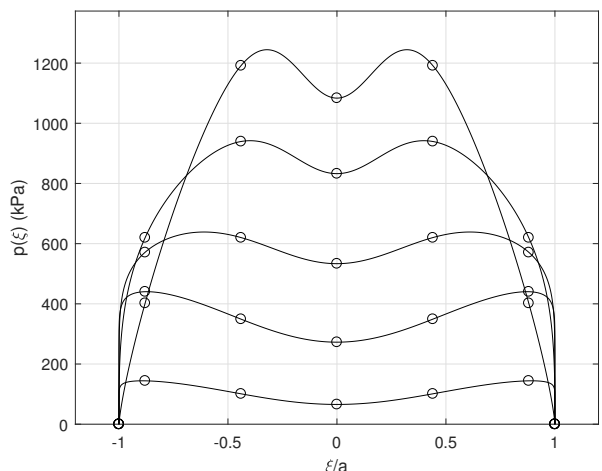

(a)

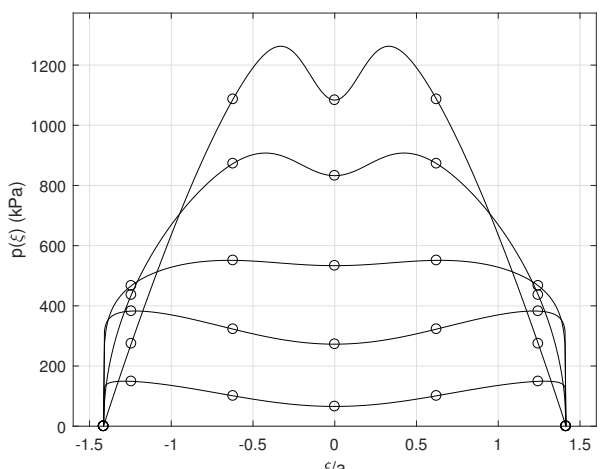

(b)

Figure 1. Data from five loading cases in the experiment of Murzenko [3]: (a) taken from pressure cells across the center of a square footing of width $2 \mathrm{a}$; (b) across the $45^{\circ}$ diagonal. The data are curve-fit using Equation (1).

\subsubsection{The Foundation-Sandpile Analogy}

There exists a number of experimental and analytical results in the field of granular physics that provides the basis for a hypothesis along these lines. It is well-known that the pressure-dip exists in normal stress distributions measured at the bases of static sandpiles. The measurements of Smid and Novosad [22] are particularly noteworthy, providing values for the shear stress components corresponding to the normal pressure fields. Comparison of these results to the foundation contact pressure measurements of [1-3] reveals a remarkable resemblance in stress fields developed in contact interfaces with a rigid structure. A series of experiments by Brockbank et al. [23] provided a strong empirical correlation between the stress state (i.e., peak pressure location) with the angle or repose of the granular material, 
which is naturally related to a soil's angle of internal friction. A further experimental work by Vanel et al. [24] produced the following striking result; the resulting stress distribution at the base of a sandpile is qualitatively dependent on its construction method. This is to say that the equilibrium state of the granular mass is fundamentally linked to its history (deposition and loading), prompting the concept of a sand's "memory." Piles formed progressively from localized sources showed the pressure dip; those formed by a transition from a uniform flowing state to a mechanically stable jammed state had parabolic stress distributions with maximum values at the center of the pile. The results were qualitatively invariant between circular and rectangular base plates. Another important finding is that the measured stress distributions within the sandpiles were shown to be largely independent of the size of the pile; the normal stress profiles strongly collapse to one distinct curve when normalized over a wide range of pile heights for the same material with localized source construction history. The role of the deposition process in sandpile stresses was later investigated by Ai et al. [25]. It has also been shown by Zuriguel and Mullin [26] that inherent soil properties such as particle shape have qualitative effects on the resulting pressure distributions.

The mechanical explanation for the stress behavior observed within sandpiles is that a natural arching effect occurs as the forces carried by the bulk material are distributed along intergranular force chains [27]. The pressures are therefore distributed and supported through a wedge of granular material by frictional force networks, which redistribute granular body forces away from the center of the pile, thus leading to the pressure dip.

A good example of the explanation of the sandpile pressure dip is the Fixed Principal Axis (FPA) theory ([28,29]). The continuum stress equilibrium equations are closed by the assumption that intergranular force chains fix the direction of the principal stress axis within the pile, providing analytic expressions of the stress tensor which depend only on the angel of repose and match very well with experiment. The FPA model stress tensor also contains a radially acting shear stress component with zero values at the edge and center of the pile and with a peak location shared with the normal distribution as shown in Figure 2. The prediction of the contact stresses matches well with the experimental sandpile results of discussed above.

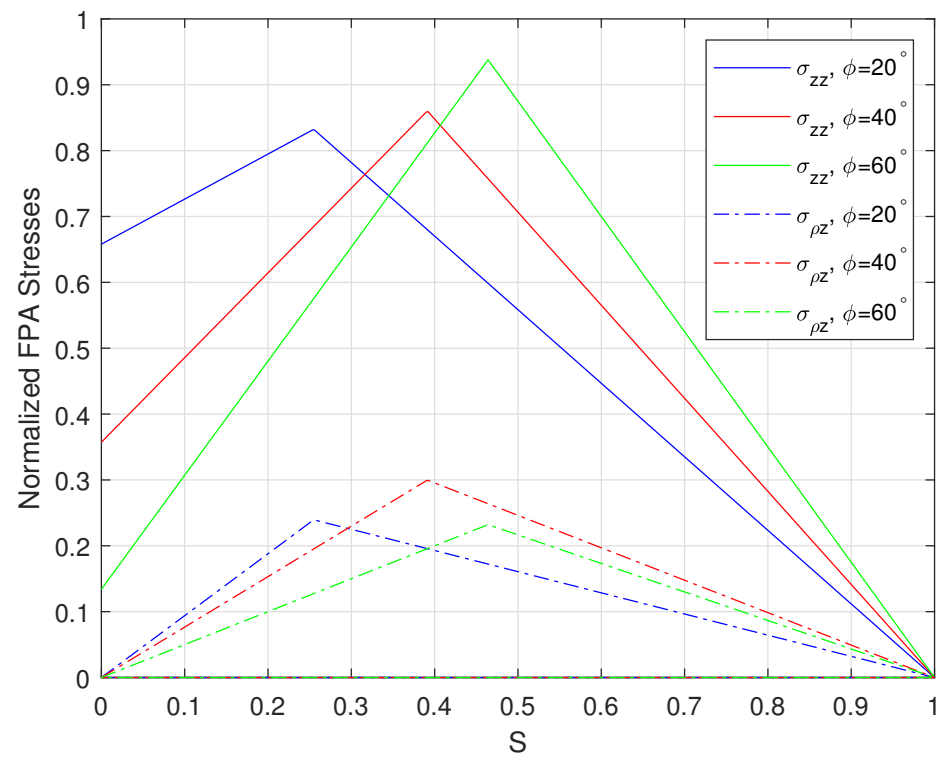

Figure 2. Representative analytical sandpile stresses from the Fixed Principal Axis model ([28,29]). Normal stress $\sigma_{z z}$ is plotted alongside the corresponding radial shear stress $\sigma_{\rho z}$ for three values of the angle of repose $\phi$; values are plotted with respect to a normalized coordinate $S=\frac{|x| \tan \phi}{z}$. Note that the location of the peak stress magnitudes is a function of $\phi$ alone. 
The pressure-dip phenomena is driven by the inherent physical mechanisms of the granular material. Furthermore, the stress phenomena measured and calculated for static sandpiles are qualitatively the same as those found in the shallow foundation contact problem. Apart from this phenomenological observation, further geometrical evidence strengthens the analogy between the two problems. It is well-known from the classical theory of foundation bearing capacity [30] that at the ultimate state of plastic failure there exits a wedge of elasticity-dominant soil immediately beneath the footing, which settles in rigid motion alongside it (Figure 3b). The existence of this wedge has been verified photographically in real soils, for example by Biarez et al. [31]. Furthermore, the wedge formation can be visualized using Finite Element Method (FEM) [32] and Discrete Element Method (DEM) [33-35] numerical analyses of shallow foundation systems. For a given load smaller than the bearing capacity, the elastic wedge begins to form as shear planes develop at angles dependent on the degree of mobilized internal friction. With increasing foundation forces this wedge evolves to that predicted at the ultimate state. The differences between the foundation and sandpile systems are as follows:

- The free-standing sandpile distributes the weight of its own body forces alone, while the resultant traction on the footing is the response to externally applied forces.

- The boundary of the sandpile is stress-free and at a state of incipient failure, while the boundary of the foundation wedge is supported and confined by the surrounding soil bed.

The result: the foundation system is statically equivalent to a sandpile, turned upside down, with additional boundary conditions (Figure 3).

Given the similarity in wedge geometry and the traction phenomenon between soil and support structure, we argue that the contact traction fields as shown in (Figure 1) are the product of the arching effect in the formation of force chains in a wedge of soil, which evolves with externally applied loads to a relatively rigid loaded footing. These normal traction distributions evolve towards a parabolic distribution as the resultant load approaches a limit state (e.g., the distribution assumed by Terzaghi [30]). It follows that there must also exist frictional stresses resisting soil movement in parallel to the soilfooting interfaces and which correspond to the normal traction distributions in predictable ways $[6,7,21]$.

Measurements of the pressure dip in the foundation problem predate the notice of this phenomena in sandpiles in the literature by a few decades at least. Given that the latter caused significant upheaval in the study of granular physics (see [36]), the mechanical analogy suggested here may have been pointed out at an earlier time. As the FPA model provides an analytical prediction of normal and tangential stress distribution for a given angle of repose, it may be possible to predict the same for a given quantity of mobilized internal friction, which will in turn be dependent on the inherent properties of the soil as well as on load history and magnitude. The development of such a model is outside of the scope of this paper. 


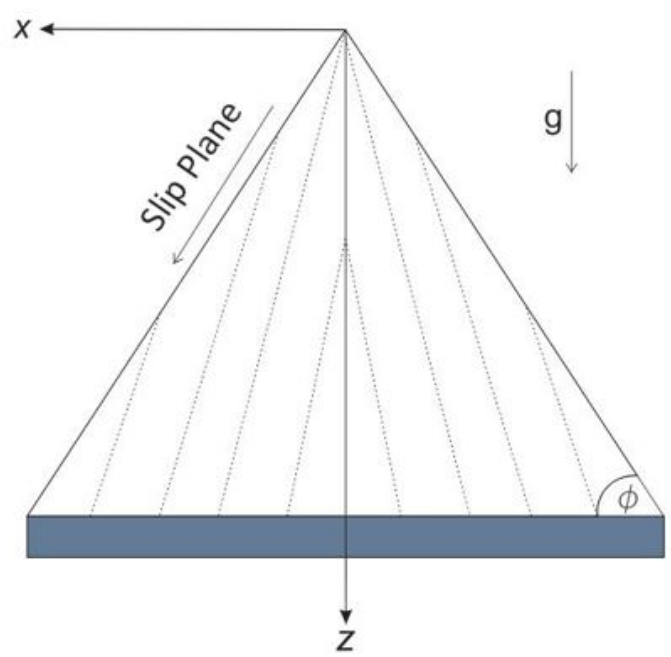

(a)

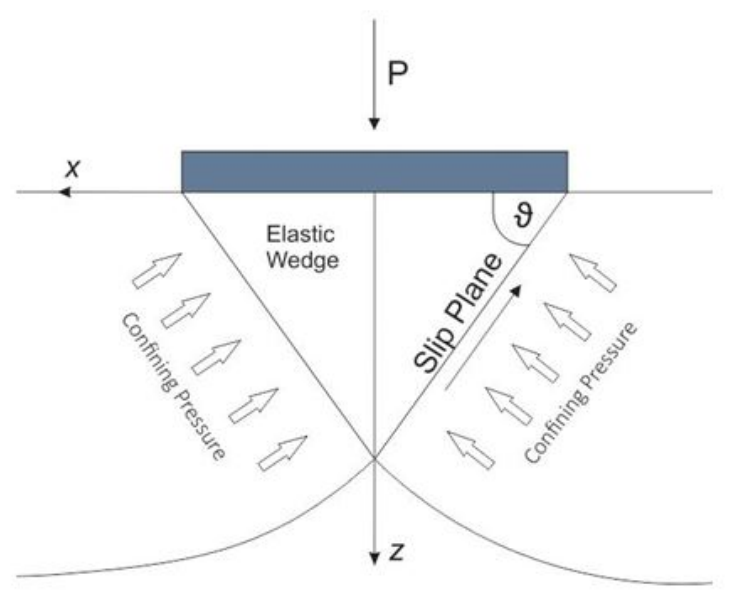

(b)

Figure 3. A qualitative comparison between the forces and geometries of (a) a static sandpile, and (b) a normally loaded shallow foundation system with underlying granular wedge. The similarities in the systems and their resulting stress phenomena (normal pressure dips and corresponding radial shear tractions fields) is used as the basis for a mechanical analogy between the two problems.

\subsubsection{Semi-Empirical Traction Functions}

A benefit of the recognition of the sandpile-foundation analogy lies in the use of semiempirical tools from one problem in the setting of another. Ai et al. [25] initially proposed an equation of the following form for the interpolation of empirical measurements of the normal compressive stresses at the bases of sandpiles:

$$
p(\xi)=A \cos \left(\frac{\pi}{2} \frac{\xi}{\alpha}\right)^{\omega}-B \exp \left(-\frac{\xi^{2}}{(\zeta \alpha)^{2}}\right) .
$$

Under the initially intended interpretation, $\alpha$ is the half-length of a sandpile and $A, B, \zeta$ and $\omega$ are curve-fit parameters. This expression was first applied to the interpolation of foundation contact traction data in [20]; it has also been used here for the curve-fits of Murzenko's data seen in Figure 1. The first term on the right-hand side of Equation (1) determines the bulk of the pressure profile, while the second term is associated with the pressure dip. Thus the parameters $A, \omega$ are associated with the magnitude and shape of the pressure, respectively. $B$ and $\zeta$ control the respective magnitude and spatial extent of the pressure dip. The parameters will ultimately depend on load magnitude, geometry, 
and history, as well as physical soil parameters, although more experimental data are necessary to correlate them with these system features. The parameters for the curve-fits of Murzenko's data in Figure 1 are given in Table 1. The five normal loading cases are from here on referred to as loads N1-N5. The subscripts 0 and 45 refer to the angle from the horizontal at which the traction measurements were measures taken across a square footing, i.e., the center line and diagonal.

Table 1. Input parameters for Equation (1) fitting the Murzenko data for loading cases N1-N5.

\begin{tabular}{ccccccccc}
\hline & $\boldsymbol{A}_{\mathbf{0}}$ & $\boldsymbol{A}_{\mathbf{4 5}}$ & $\boldsymbol{B}_{\mathbf{0}}$ & $\boldsymbol{B}_{\mathbf{4 5}}$ & $\boldsymbol{\omega}_{\mathbf{0}}$ & $\boldsymbol{\omega}_{\mathbf{4 5}}$ & $\boldsymbol{\zeta}_{\mathbf{0}}$ & $\boldsymbol{\zeta}_{45}$ \\
\hline Case N1 & 1935.557 & 2069.007 & 127.751 & 141.196 & 0.0605 & 0.0539 & 0.7367 & 0.7838 \\
Case N2 & 5819.266 & 5047.482 & 309.301 & 232.123 & 0.0619 & 0.0605 & 0.7719 & 0.8213 \\
Case N3 & 7391.370 & 6138.864 & 205.655 & 80.404 & 0.1454 & 0.1585 & 0.4888 & 0.5074 \\
Case N4 & $10,532.047$ & $10,006.803$ & 220.551 & 168.095 & 0.3166 & 0.4956 & 0.3123 & 0.2446 \\
Case N5 & $15,063.014$ & $14,008.211$ & 422.666 & 317.186 & 0.7887 & 0.9731 & 0.2785 & 0.1694 \\
\hline
\end{tabular}

A mathematical expression for the corresponding radial shear traction field was suggested in [21]. The function in that paper was able only to generate traction fields with peak locations within the inner-half radius of the sandpile or foundation. Note that all recorded sandpile data fit within this assumption; the peak location for the FPA model is also theoretically bounded within this radius. However, the empirical data from foundation measurements show that the peak location in traction can range across the entire surface of the footing (see Figure 1 for normal traction examples of his phenomenon, and [2] for tangential). This variation in the foundation traction behavior from that seen in sandpiles is likely due to the extra confinement at the boundary of the underlying soil wedge; this confining effect from the surrounding soil allows for extra support of the internal arches that redistribute the traction at the wedge base further away from the center. In order to capture the full range of phenomena, the radial-tangential traction function is modified as:

$$
q(\xi)=C \xi(\alpha-\xi) \exp \left(\frac{\left(\alpha H\left(\frac{\alpha}{2}-\xi^{*}\right)-\xi\right)^{2}}{D^{2}}\right)
$$

Here, $H(x)$ is the Heaviside step function, $\xi^{*}$ is the desired location of peak traction, and $C$ and $D$ are free parameters. It is deduced from the sandpile analogy that the peak shear traction occurs at the same location as the peak in the normal traction field; thus $\xi^{*}$ can be determined by differentiation from Equation (1). The parameters $C$ and $D$ can then be chosen to determine Equation (2) in order to cause the resulting tangential traction to share this peak location (i.e., $\left.\frac{d q}{d \xi}\right|_{\xi}=\xi^{*}=0$ ), and to enforce a desired ratio in peak normal and tangential stresses defined as $\zeta=\frac{q\left(\xi^{*}\right)}{p\left(\xi^{*}\right)}$. An example of an outcome of this procedure is shown in Figure 4. 


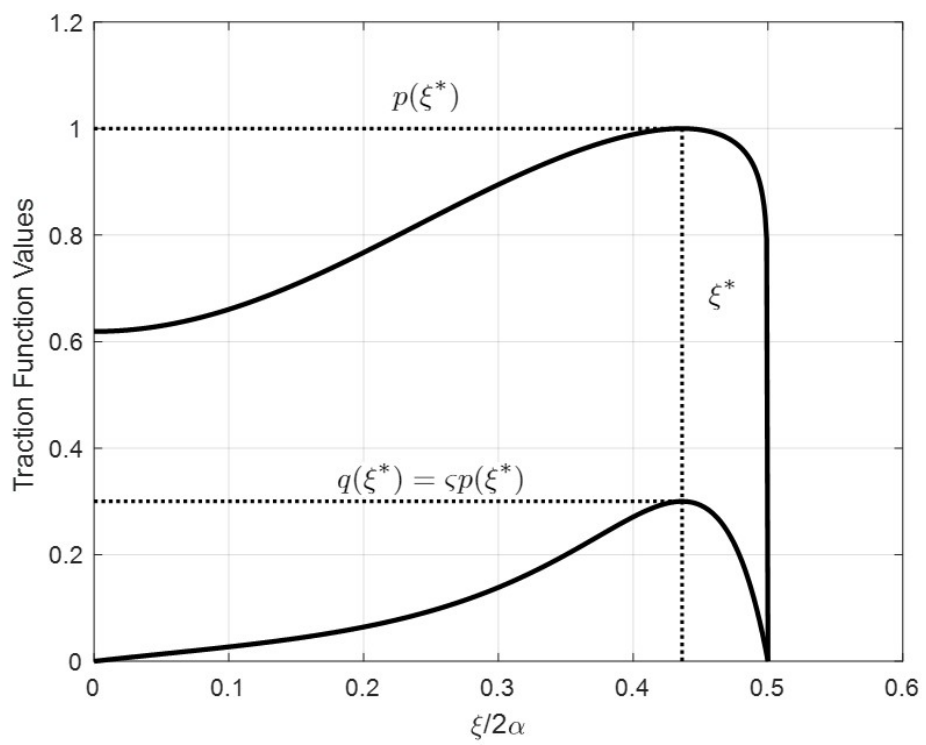

Figure 4. Example normal and shear traction curves from Equations (1) and (2), respectively.

\subsection{Parametric Study for Boundary Conditions in an Analytic Elastic Foundation Model}

It is common geotechnical practice to employ simple continuum models to approximate the settlement, stiffness, or stress states of a shallow foundation system. The classical model utilized to this effect is the homogeneous, isotropic, half-space [37-40]. This model is formulated using the elliptic partial differential equations of linear elasticity:

$$
\begin{aligned}
& (\lambda+\mu) \frac{\partial D}{\partial x}+\mu \Delta u=0 \\
& (\lambda+\mu) \frac{\partial D}{\partial y}+\mu \Delta v=0 \\
& (\lambda+\mu) \frac{\partial D}{\partial z}+\mu \Delta w=0 .
\end{aligned}
$$

Here $\lambda, \mu$ are the Lamé elastic constants, $(u, v, w)$ are the elastic displacements in the Cartesian $(x, y, z)$ directions, $D=\frac{\partial u}{\partial x}+\frac{\partial v}{\partial y}+\frac{\partial w}{\partial z}$ is the strain dilation, and $\Delta=\frac{\partial^{2}}{\partial x^{2}}$ $+\frac{\partial^{2}}{\partial y^{2}}+\frac{\partial^{2}}{\partial z^{2}}$ is the Laplacian differential operator. Historically, constant Neumann (traction) boundary conditions normal to the contact plane are assumed in order to represent the average contact pressure between a concentrically loaded rigid footing and underlying soil body [39]. In general the Cartesian components of Neumann boundary conditions are the traction fields $q_{x}, q_{y}, p$ acting in the directions $x, y, z$ on the boundary plane $z=0$. These are ultimately related to components of the stress tensor and the displacement vector $(u, v, w)$ on the boundary by Hooke's law:

$$
\begin{aligned}
& q_{x}=-\left.\sigma_{x z}\right|_{z=0}=-\mu\left(\left.\frac{\partial u}{\partial z}\right|_{z-0}+\left.\frac{\partial w}{\partial x}\right|_{z=0}\right) \\
& q_{y}=-\left.\sigma_{y z}\right|_{z=0}=-\mu\left(\left.\frac{\partial v}{\partial z}\right|_{z=0}+\left.\frac{\partial w}{\partial y}\right|_{z=0}\right) \\
& p=-\left.\sigma_{z z}\right|_{z=0}=-\left.(\lambda+2 \mu) \frac{\partial w}{\partial z}\right|_{z=0}-\lambda\left(\left.\frac{\partial u}{\partial x}\right|_{z=0}+\left.\frac{\partial v}{\partial y}\right|_{z=0}\right)
\end{aligned}
$$

In most past analyses, the tangential contact tractions $q_{x}, q_{y}$ are assumed to be zero. Because these assumptions may be inaccurate, recent work has been conducted in order to facilitate the calculation of the stress and displacements fields for this problem under arbitrary assumptions of boundary traction variation and direction. The solutions for the 
following examples are obtained using the method of superposition of interpolated closedform solutions for bilinear traction distributions as outlined by Taylor and Chung [20,21].

Given the preceding discussion of the normal and tangential boundary traction fields which develop at foundation contact interfaces, the purpose of this section is to show that the distribution and direction of the boundary traction fields have a non-negligible effect on the results of traditional elastic analysis. The application of empirical knowledge regarding these fields as boundary conditions yields results that are better in line with known experimental data. In order to model soil-structure interaction through a square footing, surface traction components take finite nonzero values inside a square region of length $2 a$ centered at the origin, as in Figure 5. The surface traction fields are assumed zero outside of this region. The coordinates $x^{\prime}, y^{\prime}$ are defined as the values of coordinates $x, y$ within this square region. Normal traction fields are selected which exhibit the pressure dip behavior in Equation (1) are employed as the boundary condition $p\left(x^{\prime}, y^{\prime}\right)$. Corresponding radially acting tangential traction fields are later prescribed as supplementary boundary conditions $q_{x}\left(x^{\prime}, y^{\prime}\right), q_{y}\left(x^{\prime}, y^{\prime}\right)$, and the effect of parametrizing the peak stress ratio $\varsigma$ of Equation (2) on the resulting displacement and stress fields is studied.

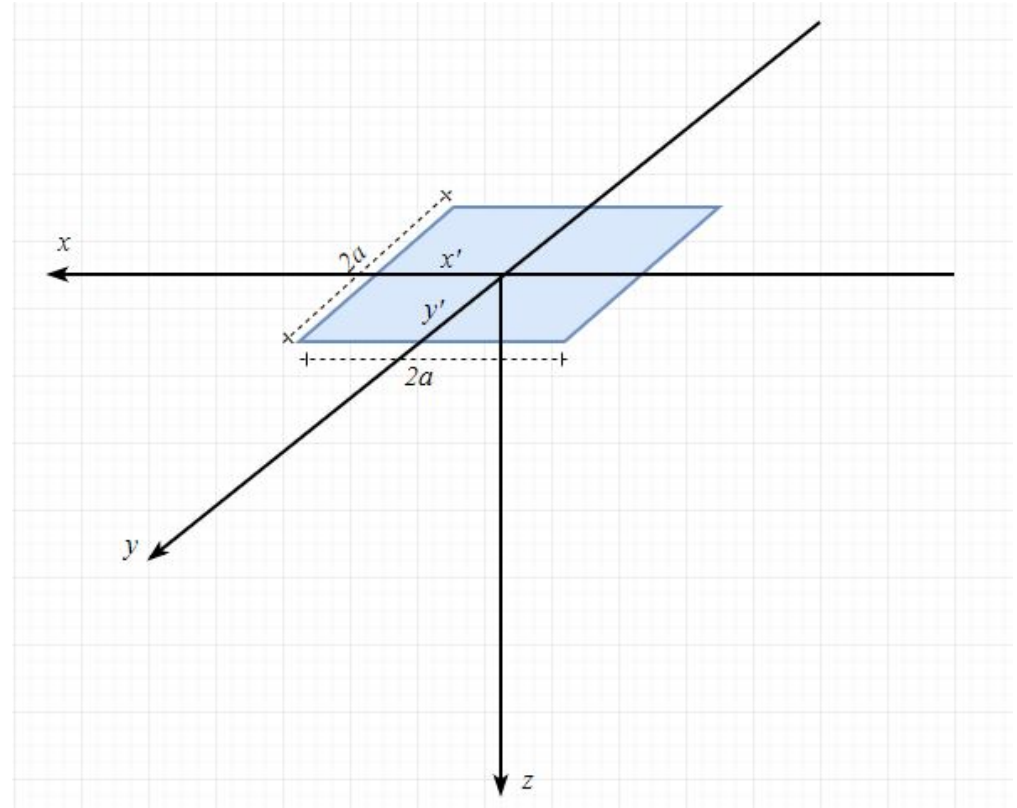

Figure 5. Geometry and coordinate system of an elastic half-space.

\subsubsection{Behavior Due to Evolving Normal Load}

The one-dimensional curve fits of Equation (1) of the five normal loading cases of a rigid square surface footing depicted in Figure 1 (from [3]) are mapped continuously to the square using a procedure outlined in $[20,21]$. The traction surfaces that result from this mapping are shown in Figure 6. These loading surfaces clearly exhibit the evolving pressure dip phenomenon. The peak traction location is analyzed with respect to total load in Figure 7. 


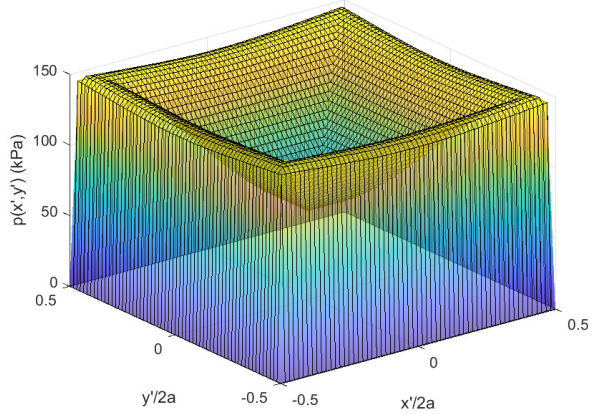

(a)

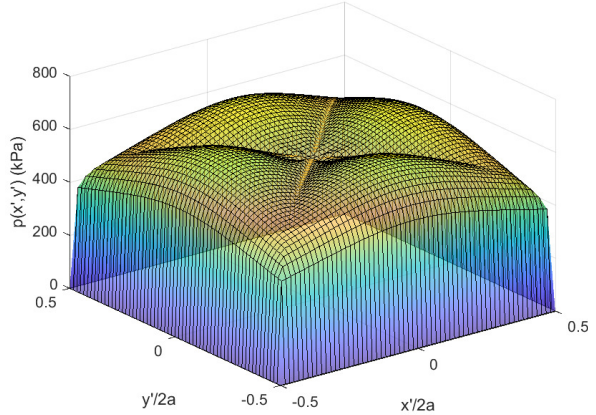

(c)

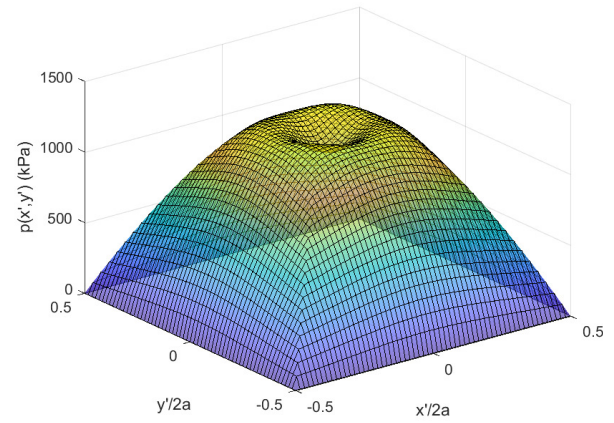

(e)

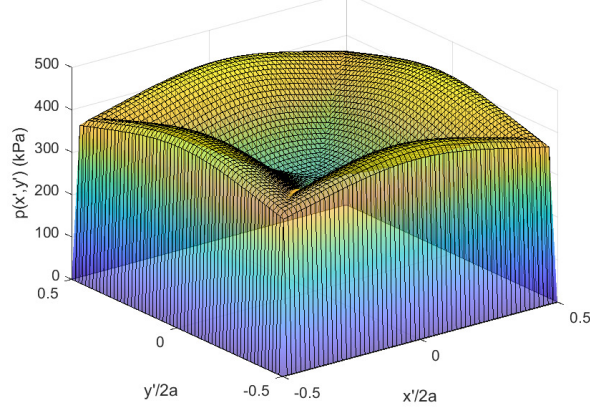

(b)

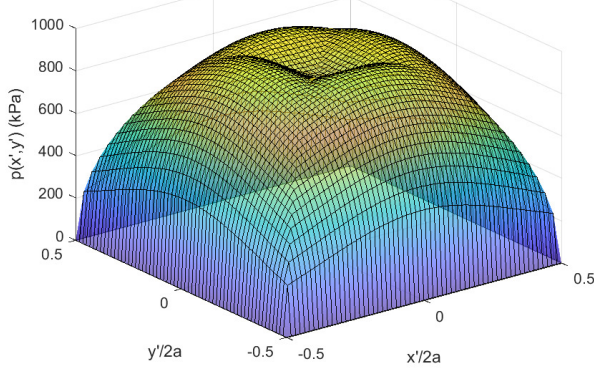

(d)

Figure 6. Normal traction surface examples mapped from Equation (1) to a square area: (a) Case N1; (b) Case N2; (c) Case N3; (d) Case N4; (e) Case N5.

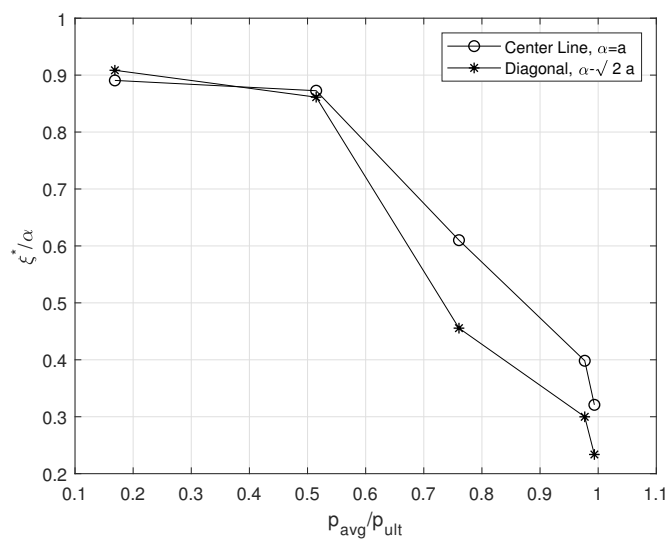

Figure 7. Analysis of peak locations versus applied average pressure from the curve-fit pressure surfaces of Equation (1). 
Taking these loading surfaces as normal boundary conditions, the effect of increasing load magnitude on a square footing resting on dry sand can be analyzed by calculating the resulting elastic displacements and stresses in the half-space. In order to isolate the influence of the evolving normal traction, it is assumed initially that $q_{x}=q_{y}=0$. Figure 8 shows the results for normalized vertical ( $z$-direction) surface displacement fields. The results are normalized by the material elastic parameters and footing size:

$$
w_{p}^{*}(x, y) \equiv \frac{E}{2 a\left(1-v^{2}\right)} w_{p}(x, y, 0),
$$

where $w_{p}$ is the component of total vertical displacement $w$ due to the normal traction $p$ alone, $E$ is Young's modulus, and $v$ the Poisson ratio. Figure 8 a shows the cross-sectional values of Equation (4) across the center line of the loaded region. Figure $8 \mathrm{~b}$ gives a better picture of the evolution of stiffness throughout the contact region, which corresponds to each loading traction distribution. The force-displacement behavior is nonlinear across the spectrum of loading cases, and this behavior varies according to the location of the point within the loaded region. This is to be expected, as the traction fields tend to concentrate towards the center of the region with increasing applied force. Correspondingly, points closer to the center experience larger displacement for the higher loading cases, while points closer to the edge appear to stiffen as the bulk of the contact traction is distributed away from these regions. Due to the linearity of the material and contact geometry of this model, it is clear that this nonlinearity stems for the evolution of the empirical contact traction fields alone. In the real granular material, however, the measured evolution in contact traction almost certainly occurred in conjunction with irreversible deformations within the granular structure. The contact nonlinearity appears slight for cases N1-N4, but is most drastic for the final case N5. The traction is redistributed to such a degree between N4 and N5 that the edge displacements calculated for the final case are larger than the former; while this phenomena is likely nonphysical, it is worth noting that case N5 is described by Murzenko as the "limiting case," and that the traction was "recorded after the soil began to 'overflow' at the surface". The load magnitude of this case is also very close to the ultimate bearing capacity calculated by the classical theory of Terzaghi [30]. Thus the nonphysical "reversal" in displacement toward the edge of the footing domain between loads N4 and N5 can be attributed to this model's consideration of only contact nonlinearity. In reality, the drastic change in contact traction between these two loads would occur in conjunction with material nonlinearity, likely including large and irreversible deformations in the form of local particle rearrangement or flow. It is interesting at least that this phenomena suggests itself in a purely elastic analysis dependent only on the empirically measure distributions of surface traction; the nonphysical limiting behavior of the edge displacements may be removed by extending this analysis by including a model for material nonlinearities. In the present model, the peak displacement value (at the origin) calculated for case N5 is almost $40 \%$ larger than that extrapolated linearly from the initial stiffness at case N1, suggesting a non-negligible influence in the distribution of surface contact traction.

Figure 9 depicts the distributions of vertical stress beneath the loaded region as it is transferred to the elastic body and dissipates with depth. The trend clearly presents itself that the wider and deeper the pressure dip in the surface traction, the further the pressure dip propagates into the body with depth. Regardless of the surface conditions, the distribution of $\sigma_{z z}$ eventually become parabolic at some value of $z$. The extent to which the pressure dip propagates beneath the contact can be compared to the measurements of [1]. That the normal stress profile will eventually become parabolic in the elastic domain is evident from comparison with the distributions beneath uniform (constant) traction distributions [20]; Saint Venant's principle guarantees that the difference in the effects of statically equivalent loads becomes negligible at large distances from the load (see [41]). However, Figure 9 shows that for cases N1-N5 (i.e., when there is zero surface shear traction), the pressure dip vanishes almost immediately beneath the applied load. 


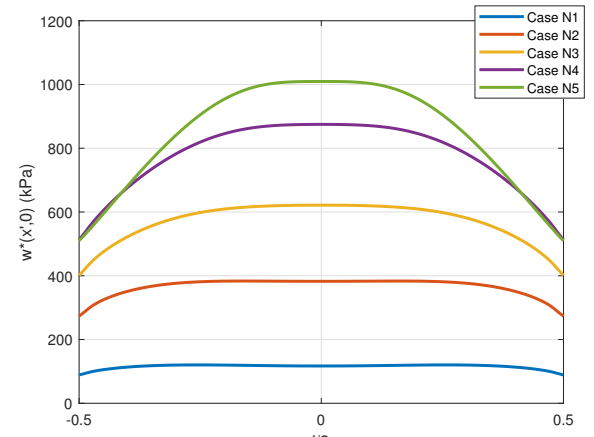

(a)

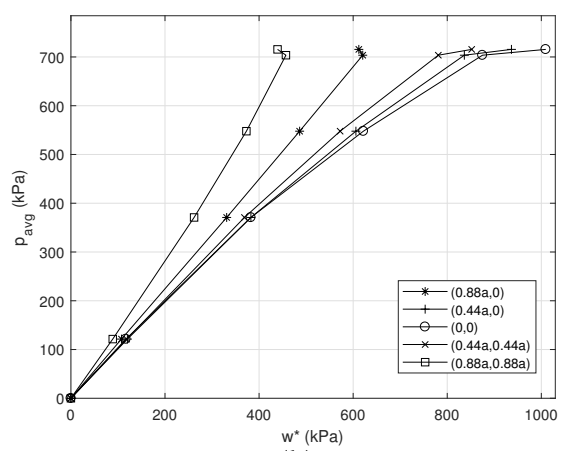

(b)

Figure 8. Results for normalized vertical surface displacement $w^{*}$ under five normal compressive traction loading cases N1-N5. (a) Cross-sectional displacement across the center of the loaded region $\left(y^{\prime}=0\right)$. (b) Average pressure vs. normalized displacement for five points within the loaded domain.

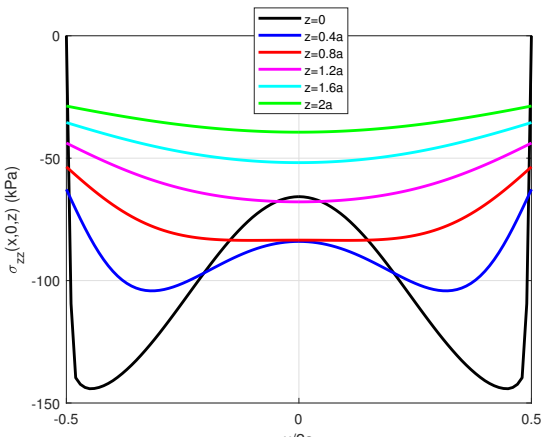

(a)

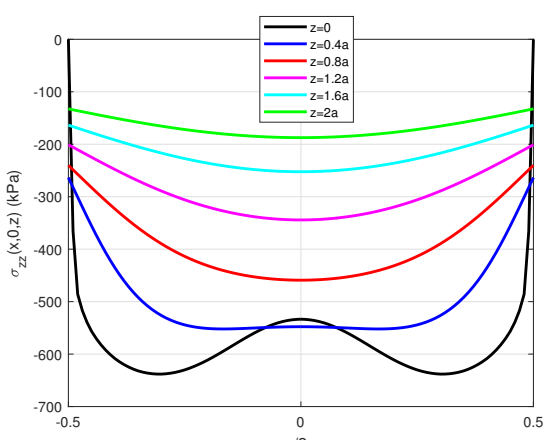

(c)

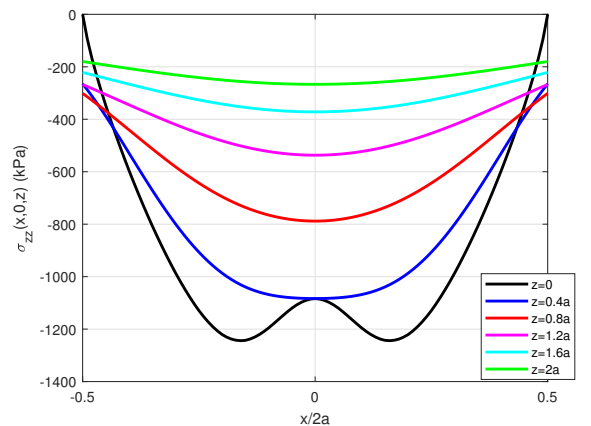

(e)

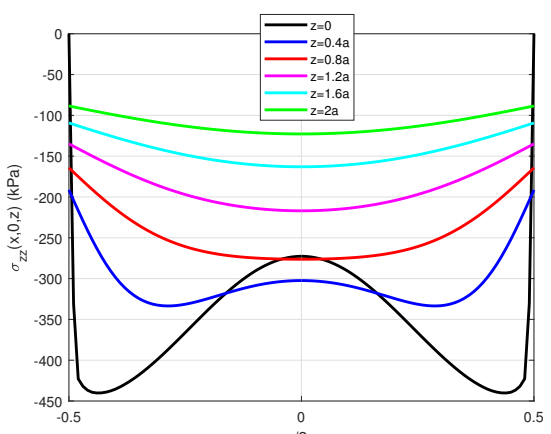

(b)

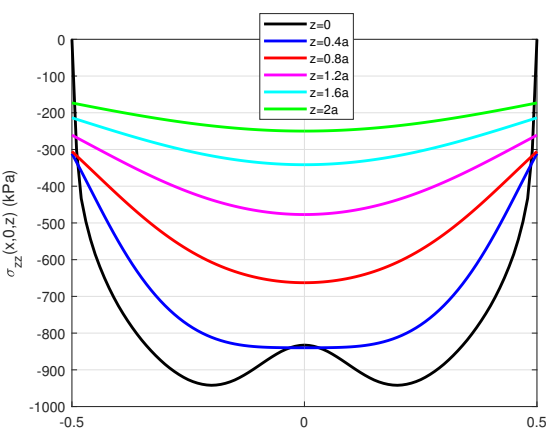

(d)

Figure 9. Vertical compressive stress $\sigma_{z z}$ in central cross sections with depth: (a) Case N1-T0; (b) Case N2-T0; (c) Case N3-T0; (d) Case N4-T0; (e) Case N5-T0. 


\subsubsection{Effects of Tangential Traction Fields}

Assuming friction at the contact interface, the normal loading cases N1-N5 will each produce a corresponding nonzero distribution of radially acting tangential traction $q_{\rho}$. These are accounted for in this analysis by prescription of auxiliary boundary conditions $q_{x}, q_{y}$ in addition to the normal traction $p$ already given. The peak locations of the tangential traction fields are assumed to correspond to that of the normal as in Figure 4; the ratio of the peak traction values $\zeta=\frac{q\left(\xi^{*}\right)}{p\left(\xi^{*}\right)}$ is assumed the same for the center and diagonal crosssections, but is taken as free parameter which accounts for the unknown relative magnitude of the tangential traction fields. The combined loading cases can therefore be specified by designation N\#-T $\varsigma$. Equation (2) is mapped to a surface traction distribution $q_{\rho}\left(x^{\prime}, y^{\prime}\right)$ in the same manner as the normal traction fields. The five tangential traction surfaces corresponding the the normal loading cases are shown in Figure 10, and the parameters for each loading case are given in Table 2. To ease analysis in Cartesian coordinates, radialtangential surface traction fields are mapped to the components of traction acting in the $x$ and $y$ directions:

$$
\begin{aligned}
& q_{x}\left(x^{\prime}, y^{\prime}\right)=\cos \left(\theta\left(x^{\prime}, y^{\prime}\right)\right) q_{\rho}\left(x^{\prime}, y^{\prime}\right) \\
& q_{y}\left(x^{\prime}, y^{\prime}\right)=\sin \left(\theta\left(x^{\prime}, y^{\prime}\right)\right) q_{\rho}\left(x^{\prime}, y^{\prime}\right) .
\end{aligned}
$$

These boundary conditions are used to analyze how the inclusion of tangential surface traction, and the manipulation of the parameter $\zeta$, effect the distributions of the elastic fields.

Table 2. Input parameters for the radial shear traction function from Equation (2), corresponding to the five normal traction loading cases (N1-N5) examined in this study. The peak traction ratio $\varsigma$ is further parametrized in order to study the effect of the magnitude of frictional shearing forces, which may develop at the contact interface.

\begin{tabular}{ccccccc}
\hline Case & $\boldsymbol{C}_{\mathbf{0}} / \boldsymbol{s}$ & $\boldsymbol{C}_{\mathbf{4 5}} / \boldsymbol{\zeta}$ & $\boldsymbol{D}_{\mathbf{0}}$ & $\boldsymbol{D}_{\mathbf{4 5}}$ & $\boldsymbol{\zeta}_{\mathbf{0}}^{*} / \boldsymbol{a}$ & $\boldsymbol{\xi}_{\mathbf{4 5}}^{*} / \sqrt{\mathbf{2} \boldsymbol{a}}$ \\
\hline 1 & 166.056 & 41.413 & 0.2356 & 0.3041 & 0.8907 & 1.2848 \\
2 & 850.628 & 474.082 & 0.2551 & 0.3774 & 0.8726 & 1.2180 \\
3 & 8091.466 & 4031.808 & 0.5741 & 1.2330 & 0.6101 & 0.6443 \\
4 & $12,180.771$ & 4437.509 & 0.5954 & 0.6060 & 0.3983 & 0.4241 \\
5 & $13,072.097$ & 4512.017 & 0.4545 & 0.5076 & 0.3209 & 0.3305 \\
\hline
\end{tabular}

The effect of the tangential surface traction fields on the normal stiffness behavior of the foundation system is nearly negligible. Figure 11a shows the normalized contributions of vertical displacement $w$ across the center of the footing due to the radial tangential traction field alone; that is,

$$
w_{q}^{*}(x, y) \equiv \frac{E}{2 a(1+v)(1-2 v)} w_{q}(x, y, 0)
$$

It is clear from comparison with Equation (4) that the total vertical displacement $w=w_{p}+w_{q}$ can only be plotted when definite values of the elastic parameters are chosen; for the sake of example, values of $E=9806.65 \mathrm{kPa}$ and $v=0.4$ are taken, being reasonable assumptions for a dense sand [42]. Figure 11b-d show the combined total displacement for varying shear ratio $s$. As the results do not vary considerably, it is reasonable to conclude that the vertical surface displacement is almost entirely dominated by the magnitude and distribution of the normal traction component. In particular, the presence of nonzero tangential traction does not remove the nonphysical overlap of the edge displacements discussed in the last section and portrayed in Figure 8. 


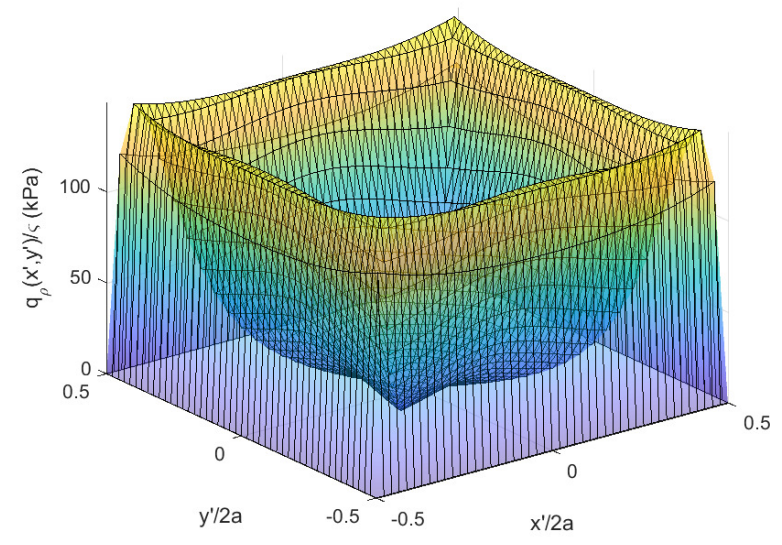

(a)

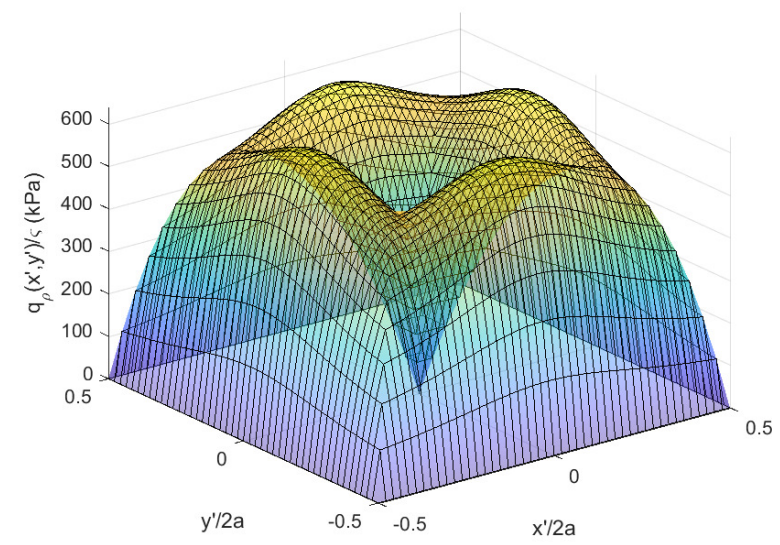

(c)

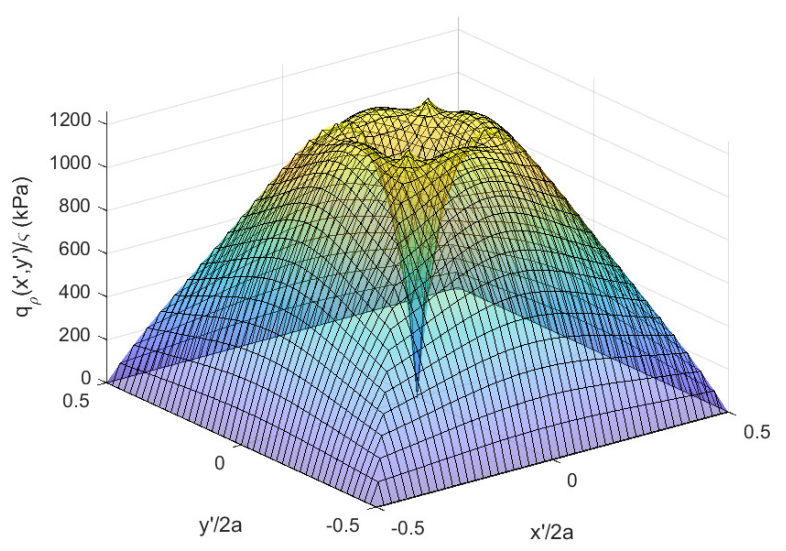

(e)

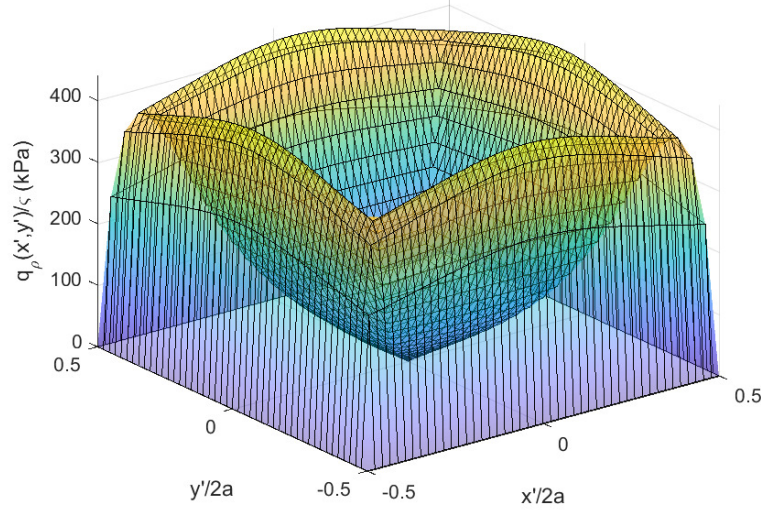

(b)

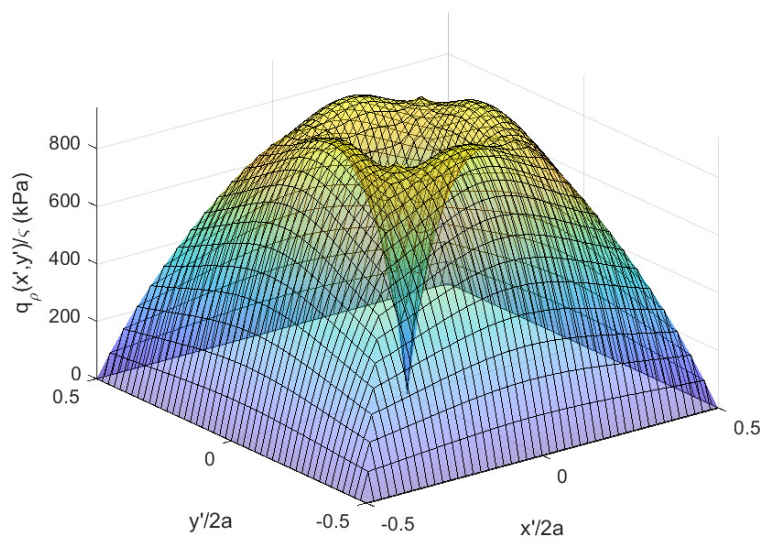

(d)

Figure 10. Radial shear traction surface examples from Equation (2) mapped to a square area. Subplots (a-e) correspond to normal loading cases N1-N5, respectively; the shear traction distributions are normalized by the peak traction ratio $\varsigma$. 
The tangential traction components have a much larger effect on the stress tensor than on the normal stiffness behavior in the elastic foundation model. Figures 12-14 show the distributions of vertical compressive stress $\sigma_{z z}$ beneath the center of the loaded surface region with increasing values of the shear ratio $\varsigma$. This analysis shows that the increased presence of frictional shear traction at the contact interface leads to an increase in magnitude and depth of the pressure dip within the elastic medium beneath the load; the values of $\sigma_{z z}$ at $z=0$ do not vary as they are fixed as boundary conditions. The experiment of Lazebnik and Smirnov [1] show that the pressure dip does in fact extend to some depth within a sand body loaded by a rigid foundation, while Figure 9 suggests that this is not the case for an elastic medium with zero tangential surface traction. The presence of friction at the contact interface, modeled via the tangential traction components, causes the spatial propagation of the pressure dip in the component of the stress tensor normal to the contact plane. The relationship between the peak shear ratio $\zeta$ and the depth of propagation of the pressure dip are studied numerically and presented in Figure 15. Given the mechanical explanation of arching within an underlying wedge of soil, these results may be correlated with the depth of the elastic wedge.

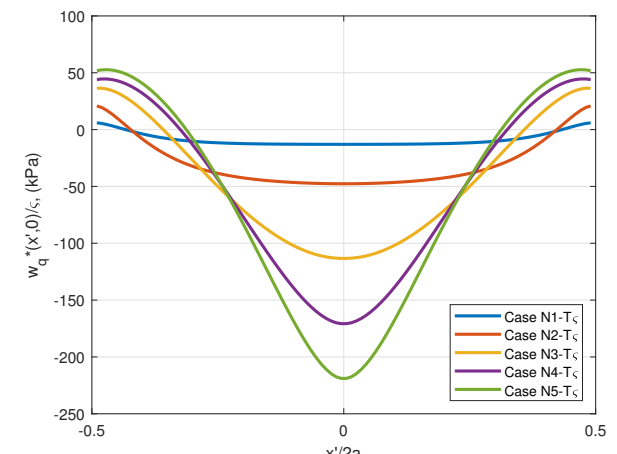

(a)

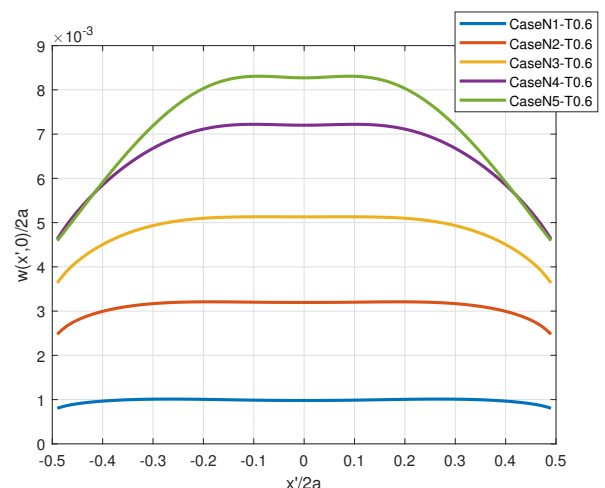

(c)

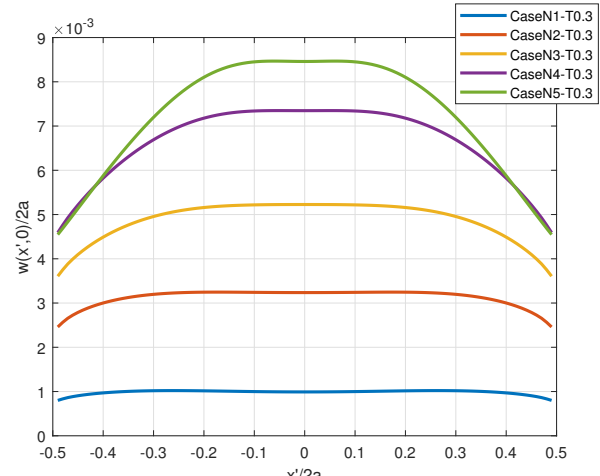

(b)

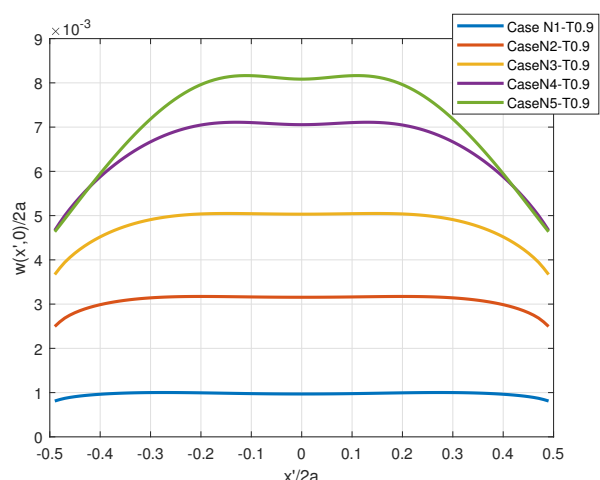

$(\mathbf{d})$

Figure 11. Effects of tangential contact traction on total vertical displacement. (a) Normalized component of vertical displacement due to the tangential traction components alone (from Equation (7)).

(b) Resulting displacements from combined normal and tangential loading cases when $\varsigma=0.3$; (c) $\varsigma=0.6$; and (d) $\varsigma=0.9$. Values of $E=9806.65 \mathrm{kPa}$ and $v=0.4$ were chosen for the combined load displacement influences in $(\mathbf{b}-\mathbf{d})$. 


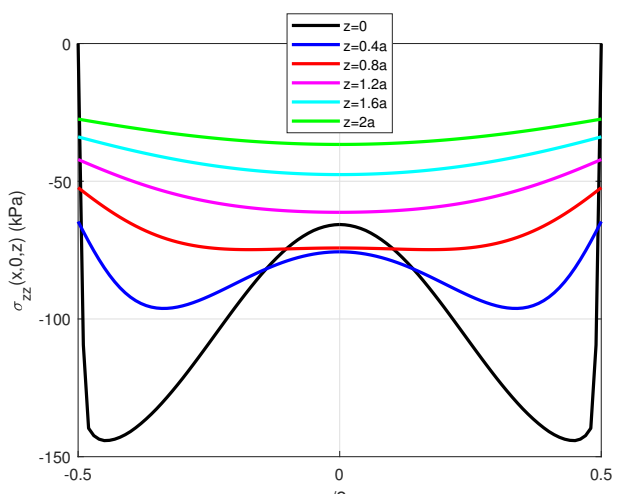

(a)

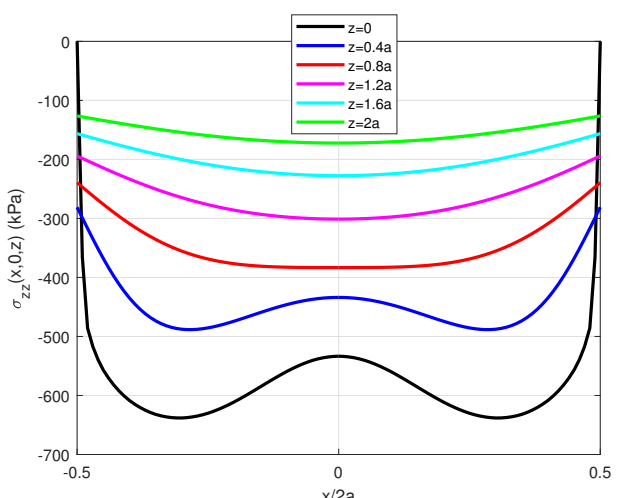

(c)

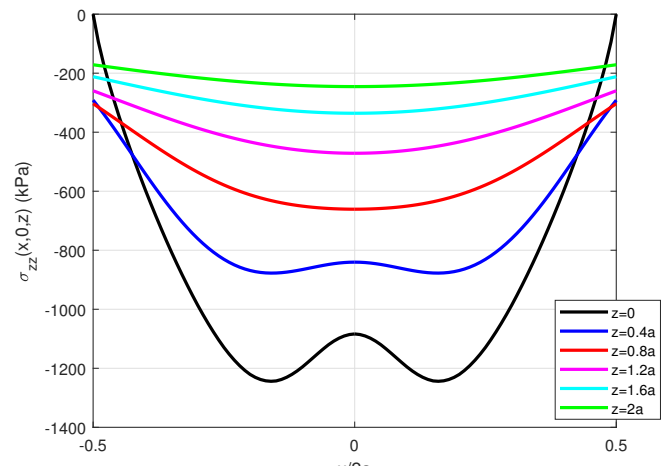

(e)

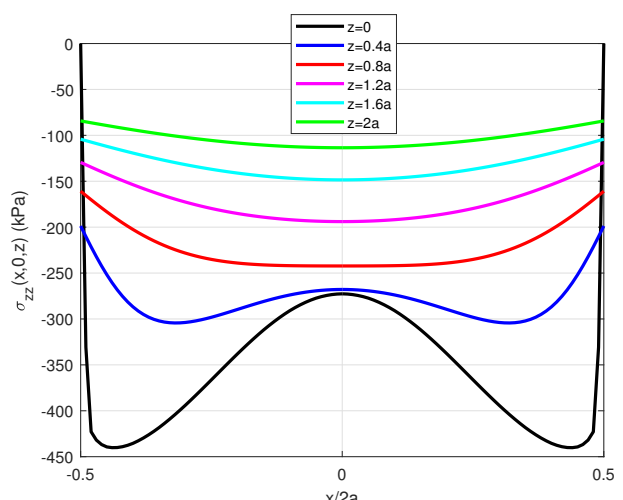

(b)

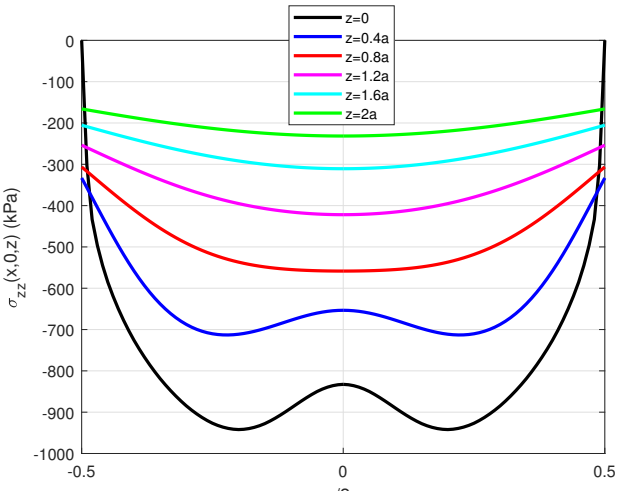

(d)

Figure 12. Vertical compressive stress $\sigma_{z z}$ in central cross sections with depth: (a) Case N1-T0.3; (b) Case N2-T0.3; (c) Case N3-T0.3; (d) Case N4-T0.3; (e) Case N5-T0.3. 


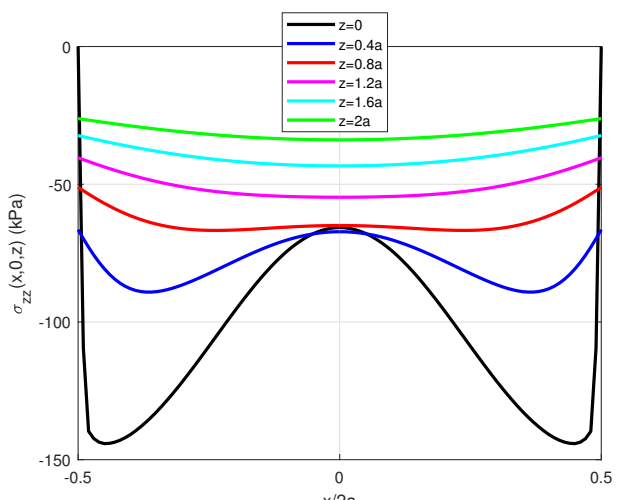

(a)

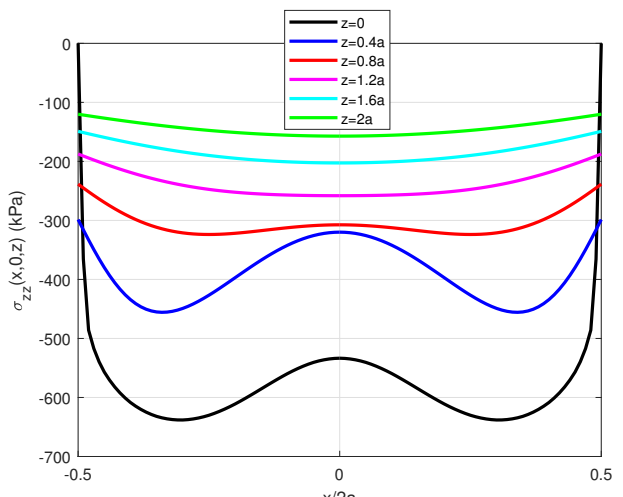

(c)

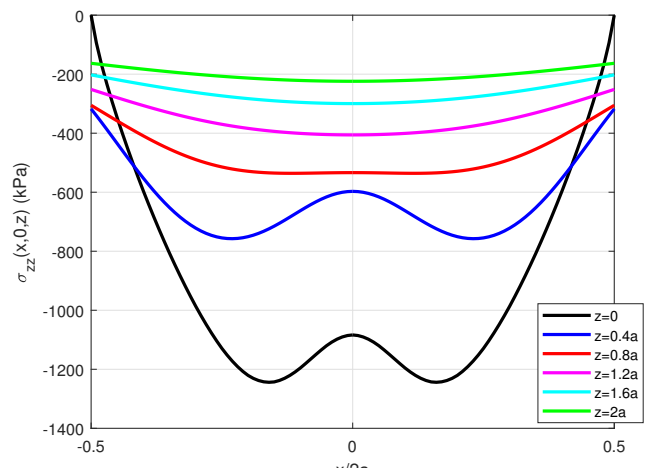

(e)

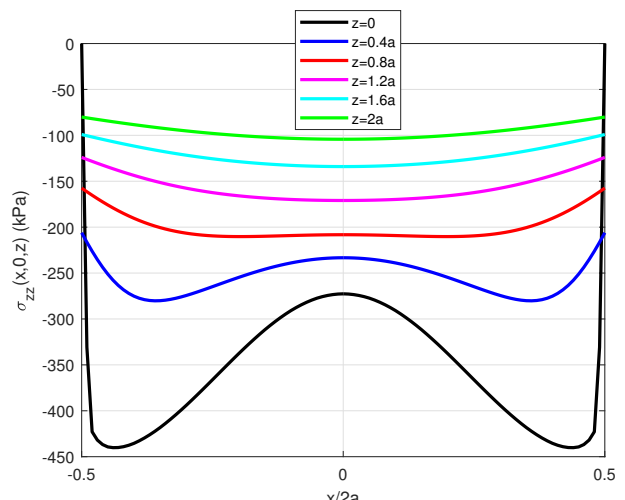

(b)

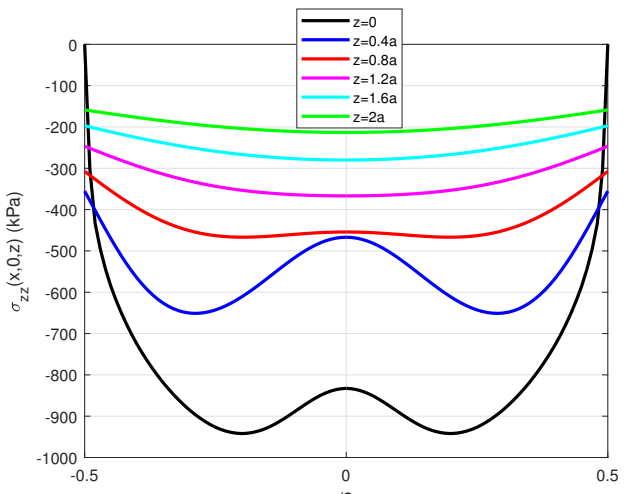

(d)

Figure 13. Vertical compressive stress $\sigma_{z z}$ in central cross sections with depth: (a) Case N1-T0.6; (b) Case N2-T0.6; (c) Case N3-T0.6; (d) Case N4-T0.6; (e) Case N5-T0.6. 


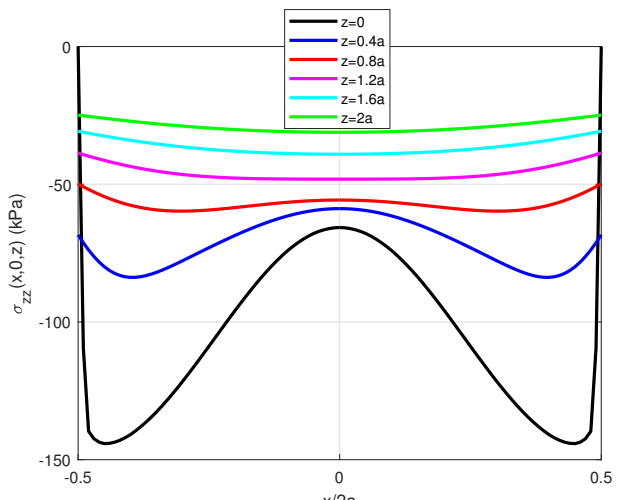

(a)

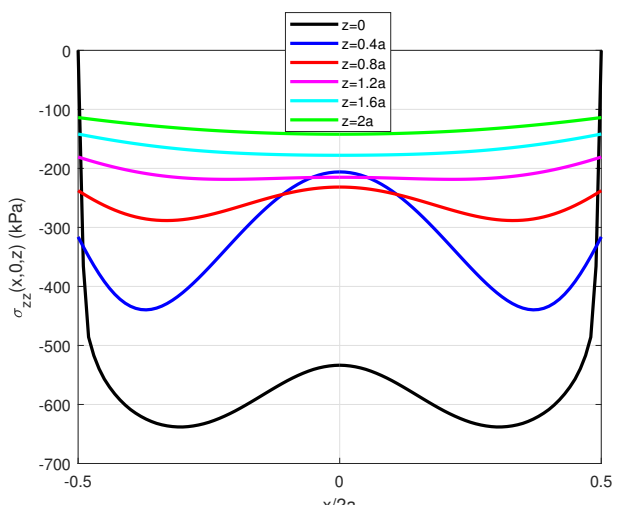

(c)

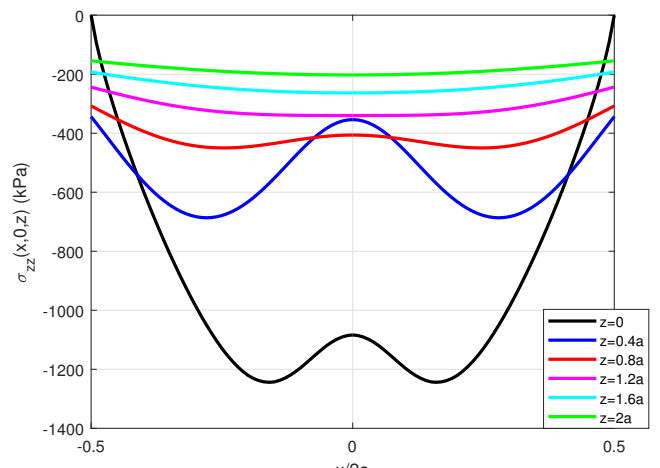

(e)

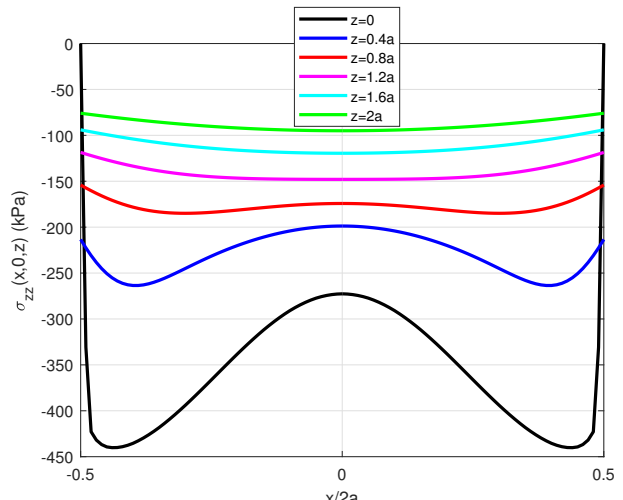

(b)

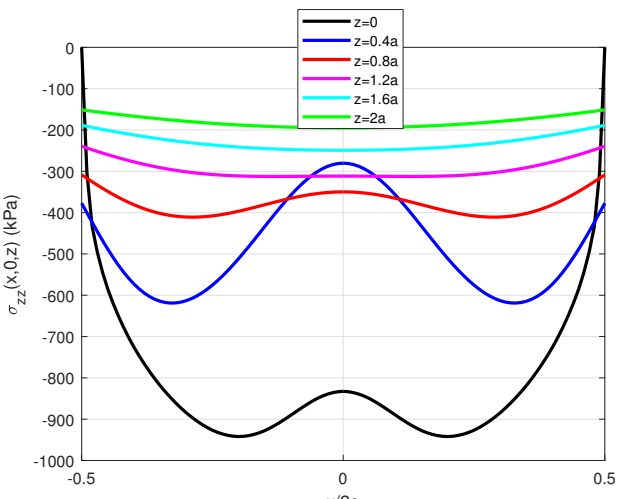

(d)

Figure 14. Vertical compressive stress $\sigma_{z z}$ in central cross sections with depth: (a) Case N1-T0.9; (b) Case N2-T0.9; (c) Case N3-T0.9; (d) Case N4-T0.9; (e) Case N5-T0.9. 


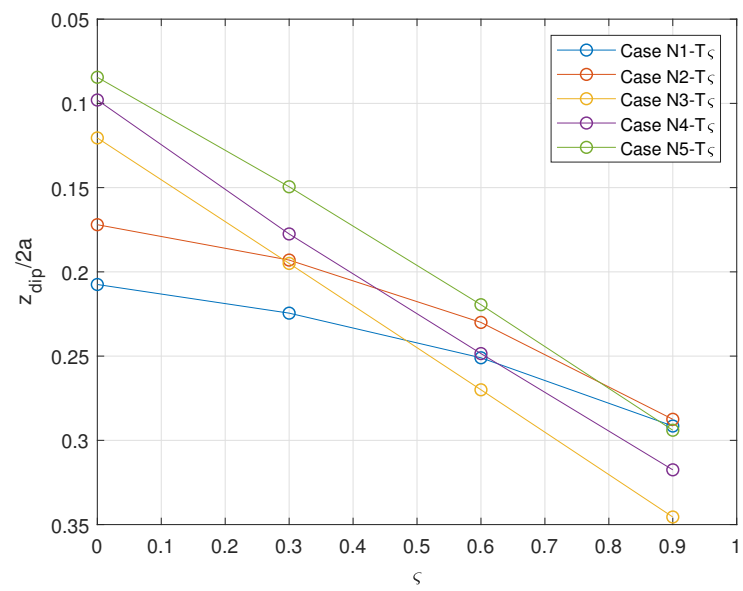

Figure 15. Propagation of the extent of the pressure dip depth $\left(z_{d i p}\right)$ in the elastic medium beneath the center of the loaded area for the five combined normal-tangential loading cases and increasing shear ratio $\zeta$.

\subsubsection{Effects of Remaining Stress Components}

The focus thus far has been on the behavior of the vertical displacement $w$ of the contact surface and the pressure dip in the stress component $\sigma_{z z}$. For the sake of completeness, a brief discussion of the remaining components of the stress tensor is provided. For the sake of brevity, this discussion is limited to the case N4-T0.3. This corresponds to the last normal loading case in which the experimental deformation remained elastic, and a physically realistic shear stress ratio, judging by the results from the FPA model (see Figure 2 or the discussion in [21]). The elastic constants are chosen again as $E=9806.65 \mathrm{kPa}$ and $v=0.4$.

The combined effects of the stress tensor are perhaps best captured by two measures; the hydrostatic pressure

$$
P=\frac{1}{3}\left(\sigma_{x x}+\sigma_{y y}+\sigma_{z z}\right)
$$

and the von Mises stress

$$
\begin{aligned}
& \sigma_{v}=\sqrt{3 J_{2}} \\
& J_{2}=\frac{1}{6}\left(\left(\sigma_{x x}-\sigma_{y y}\right)^{2}+\left(\sigma_{y y}-\sigma_{z z}\right)^{2}+\left(\sigma_{z z}-\sigma_{x x}\right)^{2}\right)+\sigma_{x y}^{2}+\sigma_{x z}^{2}+\sigma_{y z}^{2} .
\end{aligned}
$$

For comparison, similar calculations and plots were made by Forsbach [43] for normal and tangential contacts involving various axisymmetric rigid punches.

Results are compared between the "frictionless" (zero surface shear) case under load $\mathrm{N} 4$ and the case N4-T0.3. Figure 16 show a pseudocolor and contour plot of the hydrostatic pressures along the cross section $y=0$ for these two cases. Figure 17 shows the same results for the von Mises stress. The hydrostatic pressure distributions of the two cases are qualitatively similar, as the maximum pressure occurs at the surface under the load and at some distance from the center. However, the presence of the tangential surface traction tends to pull the surface maximum pressure further from the center of the loaded and region. Furthermore, these peaks are more localized and seem to move inwards towards the center with depth. This matches both what was shown in Figure 12 for the vertical stress and mimics the behavior of the arching phenomenon in a granular wedge.

Comparison between the von Mises stresses shows a much larger qualitative discrepancy. The frictionless case has peak von Mises stress at some depth beneath the boundary. The tangential traction boundary conditions tend to localize the maximum values of $\sigma_{v}$ at the boundary and towards the center of the load. The cause of this is two-fold; (i) the nonzero shear stresses provided by the auxiliary boundary conditions add large surface 
contributions when squared as per the terms in $J_{2}$ in Equation (9); (ii) there exists a small shallow region near the origin in which the horizontal stresses $\sigma_{x x} \sigma_{y y}$ are in tension, leading the difference terms $\left(\sigma_{y y}-\sigma_{z z}\right)^{2}$ and $\left(\sigma_{y y}-\sigma_{z z}\right)^{2}$ in Equation (9) to be locally large. This small tensile zone is an artifact of the outward-radially acting shear traction fields and the approximation of a soil body as an elastic continuum. As granular materials cannot sustain tension, this highlights a limitation of the elliptic Equation (3) to analyze soil-structure interaction; however, the small tensile zones can be interpreted in terms of the increased resistance due to the frictional contact and arching phenomena inside a real elastic soil body.

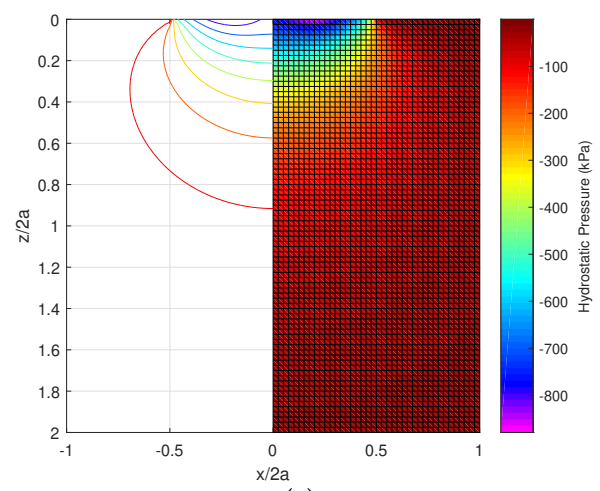

(a)

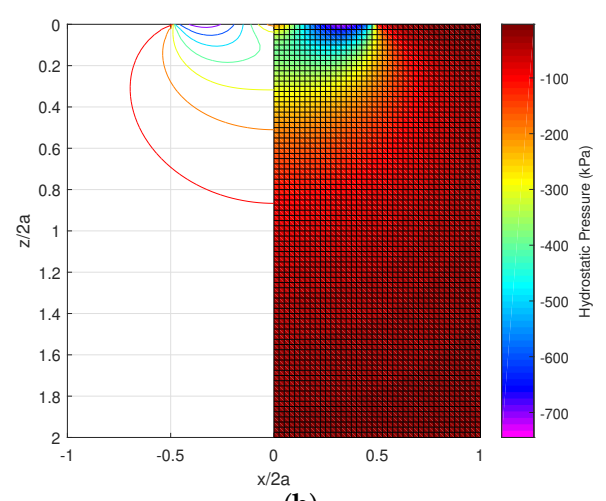

(b)

Figure 16. Hydrostatic pressure $P$ in the $x z$-plane beneath loading cases (a) N4-T0 (frictionless) and (b) N4-T0.3.

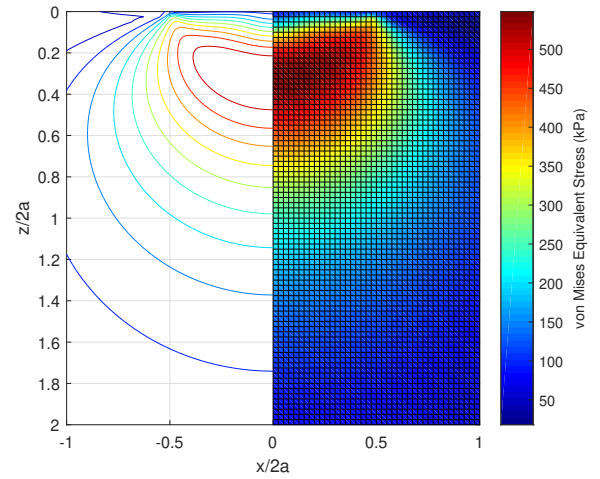

(a)

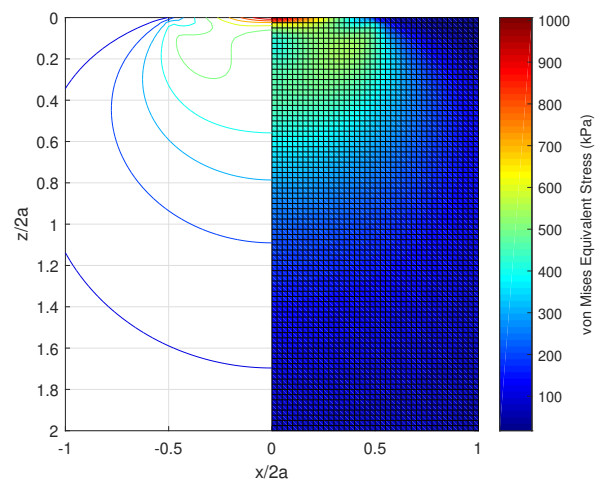

(b)

Figure 17. von Mises equivalent stress $\sigma_{v}$ in the $x z$-plane beneath loading cases (a) N4-T0 (frictionless) and (b) N4-T0.3.

\section{Discussion}

The distributions of contact stress fields on loaded foundations have been studied in past geotechnical literature primarily out of concern for quantifying the reaction forces on footings for design purposes. However, qualitatively similar phenomena in the stress fields beneath static sandpiles have been studied in the granular physics literature in the context of a fundamental aspect of the mechanical behavior of granular materials. The primary goal of the present paper has been to introduce the latter interpretation to the problem of a loaded surface footing in static equilibrium with an underlying sand, interpreted as an upside-down sandpile with lateral support. An auxiliary goal has been to show that the inclusion of these types of (normal and tangential) contact traction fields as boundary conditions can improve the results of continuum models of shallow foundation systems.

The evolution of the pressure dip in the normal traction fields recorded by Murzenko [3] and others corresponds intuitively with the reduction in stiffness with increasing load which is typical in the force-displacement behavior of shallow foundations. It has been demonstrated that the employment of a priori expressions of this traction evolution as 
boundary conditions is capable of (qualitatively) representing these phenomena in a static, purely elastic domain. In the present examples, a sharp reduction of stiffness at the center of the loaded region (and an increase at the edges) corresponds to the case where shear failure was observed beneath the foundation in the source experiment.

The inclusion of nonzero shear traction components in the boundary conditions results in a minimal increase in the foundation stiffness. However, the existence of tangential surface traction fields tends to deepen the extent to which the pressure dip exists in the normal (vertical) stress distribution with depth beneath the loaded boundary. Parametrization of the magnitude of the radial shear boundary traction component therefore provides a means to simulate the effects of body force and friction effects in a granular mass per calibration of multi-scale contact phenomena in the foundation system.

It is noted that the calculation procedure introduced here is not intended to replace more sophisticated (and expensive) numerical analyses. A sufficiently large DEM model (with the right intergranular contact/friction models and sample preparation) may be able to predict physically accurate contact pressure distributions of a footing-soil contact on a given soil while capturing internal stresses and displacement all at once; however, the authors are not aware of any case in the literature which shows the resulting normal and tangential traction fields from such an approach. Furthermore, a fully 3D model with accurate particle size and soil dimensions would be prohibitively computationally expensive for nearly all researchers and engineers. It remains an open question whether any continuum-based constitutive model could predict the correct displacements alongside the traction fields with the pressure dip. The FPA model is a static stress theory with no displacements or external loads.

The example provided here was for a homogeneous, dry, compacted sand, as reflected in Murzenko's experiment from which the boundary data were derived. Friction enhances elasticity in granular solids [44], and for that reason the simplifying (elastic) assumption was reasonable for this case, but may be less so in other scenarios. Other methods have been suggested to the authors for estimation of the response of softer and more complex soils [45]. It is currently unclear how the present approach may handle heterogeneous soil mixtures or instances where soil improvement methods have been used [46]. The effectiveness of the method depends on the measurement of boundary traction in real soils, and the extent to which these fields convey information regarding the mechanical response of the soil-structure interaction. In general, further experiments are needed in which the surface contact traction is measured at the interface between either real or model footings providing different loading conditions to different soil bodies. Furthermore, the authors are not aware of any existing experimental results which report both the measured contact traction fields alongside the resulting displacement of the footing; this type of data is required in order to fully validate the types of boundary value models discussed above.

\section{Materials and Methods}

The conclusions in the present paper are all based on data available in the cited literature. The boundary conditions are prepared using the equations and data herein and in $[3,20,21]$. The numerical results are obtained using the analytic element-based method described in [20,21]; code to implement this method is found in the supplementary material attached to these references.

Author Contributions: Conceptualization, A.G.T. and J.H.C.; methodology, A.G.T. and J.H.C.; software, A.T; validation, A.G.T. and J.H.C.; formal analysis, A.G.T.; investigation, A.G.T. and J.H.C.; resources, A.G.T. and J.H.C.; data curation, A.G.T. and J.H.C.; writing-original draft preparation, A.G.T.; writing - review and editing, A.G.T. and J.H.C.; visualization, A.G.T.; supervision, J.H.C.; project administration, J.H.C. All authors have read and agreed to the published version of the manuscript.

Funding: This research received no external funding.

Institutional Review Board Statement: Not applicable. 
Informed Consent Statement: Not applicable.

Data Availability Statement: Not applicable.

Conflicts of Interest: The authors declare no conflict of interest.

\author{
Abbreviations \\ FEM Finite Element Method \\ DEM Discrete Element Method \\ FPA Fixed Principal Axis
}

The following abbreviations are used in this manuscript:

\title{
References
}

1. Lazebnik, G.E.; Smirnov, A.A. Measurement of stresses under a rigid foundation block in the field. Soil Mech. Found. Eng. 1964, 1, 71-78. [CrossRef]

2. Muhs, H.; Bub, H. On the measurement of friction in the base of flat footings founded in sand and first test results gained by a new gauge developed for the combined measurement of normal and tangential stresses. In Proceedings of the 6th International Conference of Soil Mechanics and Foundation Engineering, Montreal, QC, Canada, 8-15 September 1965; University of Toronto Press: Toronto, Canada, 1965.

3. Murzenko, Y. Experimental results on the distribution of normal contact pressure on the base of a rigid foundation resting on sand. Soil Mech. Found. Eng. 1965, 2, 69-73. [CrossRef]

4. Ho, M.; Lopes, R. Contact pressure of a rigid circular foundation. J. Soil Mech. Found. Div. Proc. Am. Soc. Civ. Eng. 1969, 93, 791-802. [CrossRef]

5. Schultze, E. Distribution of stress beneath a rigid foundation. In Proceedings of the 5th International Conference on Soil Mechanics and Foundation Engineering, Paris, France, 17-22 July 1961; pp. 807-813.

6. Sedykh, E. On the diagram or reaction pressures under the base of a rigid foundation. Soil Mech. Found. Eng. 1964, 3, 153-157. [CrossRef]

7. Gorbunov-Possadov, M. Calculations for the stability of a sand bed by a solution combining the theories of elasticity and plasticity. In Proceedings of the Sixth International Conference on Soil Mechanics and Foundation Engineering, Montreal, QC, USA, 8-15 September 1965; Volume 2, pp. 51-55.

8. Smoltczyk, H. Stress computation in soil media. J. Soil Mech. Found. Div. 1967, 93, 101-124. [CrossRef]

9. Kerr, A. Tests and analyses of footings on a sand base. Soils Found. 1989, 29, 83-94. [CrossRef]

10. Nwabuokei, S. Measurements of Normal and Shear Stresses at the Base of a Rigid Footing Founded on Quartz Sand. Master's Thesis, University of Ottawa, Ottawa, ON, Canada, 1976.

11. Bauer, G.; Shields, D.; Scott, J.; Nwabuokei, S. Normal and shear stress measurements on a strip footing. Can. Geotech. J. 1979, 16, 177-189. [CrossRef]

12. Balakrishna, C.; Murthy, B.; Nagaraj, T. Stress distribution beneath rigid circular foundations on sands. Int. J. Numer. Anal. Methods Geomech. 1992, 16, 65-72. [CrossRef]

13. Abdullah, W. New elastoplastic method for calculating the contact pressure distribution under rigid foundations. Jordan J. Civ. Eng. 2008, 2, 71-89.

14. Harding, J.; Sneddon, I. The elastic stresses produced by the indentation of the plane surface of a semi-infinite elastic solid by a rigid punch. In Mathematical Proceedings of the Cambridge Philosophical Society; Cambridge University Press: Cambridge, UK, 1945; Volume 41, pp. 16-26.

15. Sneddon, I. Boussinesq's problem of a flat-ended cylinder. Math. Proc. Camb. Philos. Soc. 1946, 42, 29-39. [CrossRef]

16. Winkler, E. Die Lehre von der Elastizitat und Festigkeit; Dominicus: Prague, Czech Republic, 1867.

17. Johnson, K. Contact Mechanics; Cambridge University Press: Cambridge, UK, 1985.

18. Hartikainen, J. The distribution and direction of contact pressure under a rigid foundation. Acta Polytech. Scand. Civ. Eng. Build. Constr. 1972, 74, 9 .

19. Schweickert, K. Über den Einfluss von Reibungskräften in der Sohlfuge eines starren Streifenfundamentes auf die Sohldruckverteilung; Herr Klaus Boettcher: Karlsruhe, Germany, 1964.

20. Taylor, A.G.; Chung, J.H. Application of low-order potential solutions to higher order vertical traction boundary problems in an elastic half-space. R. Soc. Open Sci. 2018, 5, 180203. [CrossRef] [PubMed]

21. Taylor, A.G.; Chung, J.H. Analysis of tangential contact boundary value problems using potential functions. R. Soc. Open Sci. 2019, 6, 182106. [CrossRef]

22. Smid, J.; Novosad, J. Pressure distribution under heaped bulk solids. In Proceedings of the 1981 PowTech Conference, Institution of Chemical Engineers, Birmingham, UK, 10-13 March 1981; Volume 63, pp. D3V 1-12.

23. Brockbank, R.; Huntley, J.; Ball, R. Contact force distribution beneath a three-dimensional granular pile. J. Phys II 1997, 7, 1521-1532. [CrossRef] 
24. Vanel, L.; Howell, D.; Clark, D.; Behringer, R.P.; Clement, E. Memories in sand: Experimental tests of construction history on stress distributions under sandpiles. Phys. Rev. E 1999, 60, R5040-R5043. [CrossRef] [PubMed]

25. Ai, J.; Ooi, J.; Chen, J.; Rotter, J.; Zhong, Z. The role of deposition process on pressure dip formation underneath a granular pile. Mech. Mater. 2013, 66, 160-171. [CrossRef]

26. Zuriguel, I.; Mullin, T. The role of particle shape on the stress distribution in a sandpile. Proc. R. Soc. A Math. Phys. Eng. Sci. 2008, 464, 99-116. [CrossRef]

27. Edwards, S.; Mounfield, C. A theoretical model for the stress distribution in granular matter. III. Forces in sandpiles. Phys. A Stat. Mech. Its Appl. 1996, 226, 25-33. [CrossRef]

28. Wittmer, J.; Claudin, P.; Cates, M.; Bouchard, J. An explanation for the central stress minimum in sand piles. Nature 1996, 382, 336-338. [CrossRef]

29. Wittmer, J.; Cates, M.; Claudin, P. Stress propagation and arching in static sandpiles. J. Phys. I 1997, 7, 39-80. [CrossRef]

30. Terzaghi, K. Theoretical Soil Mechanics; John Wiley and Sons, Inc.: New York, NY, USA, 1943.

31. Biarez, J.; Burel, M.; Wack, B. Contribution a l'etude de la force portante des fondations. In Proceedings of the 5th International Conference on Soil Mechanics and Foundation Engineering, Paris, France, 17-22 July 1961; Volume 1, p. 603.

32. Abd El Samee, W.N. Effect of Shape and Depth of Shallow Foundations on Failure Mechanism and Wedge Angle of Sandy Soil. In Advanced Research on Shallow Foundations; Shehata, H., Das, B., Eds.; Springer International Publishing: Cham, Switzerland, 2019; pp. 197-216.

33. Li, N.; Cheng, M. Discrete Element Modeling on Bearing Capacity Problems. Int. J. Geol. Environ. Eng. 2016, 10, 618-625.

34. Li, L.; Jiang, M.; Li, T.; Chen, S. DEM Analyses of the Whole Failure Process of Shallow Foundation in Plate Load Test on Dense Sand. 2018. Available online: https://www.researchgate.net/publication/327208070_DEM_analyses_of_the_whole_failure_ process_of_shallow_foundation_in_plate_load_test_on_dense_sand (accessed on 8 November 2021).

35. da Rocha, J.; de Faria, M.; Albuquerque, B.; Neto, J. Shallow foundation analysis using the discrete element method. Mater. Sci. Eng. Int. J. 2019, 3, 136-139.

36. Watson, A. Searching for the sand-pile pressure dip. Science 1996, 273, 579-580. [CrossRef]

37. Boussinesq, J. Application des Potentials a L'etude de L'equilibre et du Mouvement des Solides Elastiques; Gauthier-Villars: Paris, France, 1885.

38. Love, A.E.H. The stress produced in a semi-infinite solid by pressure on part of the boundary. Philos. Trans. R. Soc. London. Ser. A Contain. Pap. A Math. Phys. Character 1929, 228, 377-420.

39. Newmark, N.M. Simplified computation of vertical pressures in elastic foundations. In Report, Engineering Experiment Station; University of Illinois at Urbana-Champaign: Champaign, IL, USA, 1935.

40. Davis, R.O.; Selvadurai, A.P.S. Elasticity and Geomechanics; Cambridge University Press: Cambridge, UK, 1996.

41. von Mises, R. On Saint Venant's principle. Bull. AMS 1945, 51, 555-562. [CrossRef]

42. Lambe, T.; Whitman, R. Soil Mechanics; John Wiley \& Sons: Hoboken, NJ, USA, 2008.

43. Forsbach, F. Stress Tensor and Gradient of Hydrostatic Pressure in the Half-Space Beneath Axisymmetric Bodies in Normal and Tangential Contact. Front. Mech. Eng. 2020, 6, 39. [CrossRef]

44. Goldenberg, C.; Goldhirsch, I. Friction enhances elasticity in granular solids. Nature 2005, 435, 188-191. [CrossRef]

45. Güllü, H.; Fedakar, H.I. On the prediction of unconfined compressive strength of silty soil stabilized with bottom ash, jute and steel fibers via artificial intelligence. Geomech. Eng. 2017, 12, 441-464. [CrossRef]

46. Çanakçi, H.; Güllü, H.; Dwle, M.I.K. Effect of Glass Powder Added Grout for Deep Mixing of Marginal Sand with Clay. Arab. J. Sci. Eng. 2018, 43, 1583-1595. [CrossRef] 